\title{
Quantum Disordered Regime and Spin Gap in the Cuprate Superconductors
}

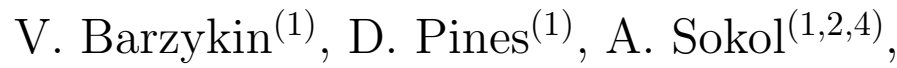 \\ and D. Thelen ${ }^{(1,3)}$ \\ (1) Department of Physics, ${ }^{(2)}$ Materials Research Laboratory, \\ and ${ }^{(3)}$ Science and Technology Center for Superconductivity, \\ University of Illinois at Urbana-Champaign, Urbana, IL 61801 \\ (4) L.D. Landau Institute for Theoretical Physics, Moscow, Russia
}

\begin{abstract}
We discuss the crossover from the quantum critical, $z=1$, to the quantum disordered regime in high- $\mathrm{T}_{c}$ materials in relation to the experimental data on the nuclear relaxation, bulk susceptibility, and inelastic neutron scattering. In our scenario, the spin excitations develop a gap $\Delta \sim 1 / \xi$ well above $\mathrm{T}_{c}$, which is supplemented by the quasiparticle gap below $\mathrm{T}_{c}$. The above experiments yield consistent estimates for the value of the spin gap, which increases as the correlation length decreases.
\end{abstract}

PACS: 74.65.+n, 75.40.Cx, 75.40.Gb, 76.60.-k 
The underdoped cuprates $\mathrm{YBa}_{2} \mathrm{Cu}_{3} \mathrm{O}_{6.63}, \mathrm{YBa}_{2} \mathrm{Cu}_{4} \mathrm{O}_{8}$, and $\mathrm{La}_{1.85} \mathrm{Sr}_{0.15} \mathrm{CuO}_{4}$, display strikingly different magnetic behavior from that measured for the fully doped system, $\mathrm{YBa}_{2} \mathrm{Cu}_{3} \mathrm{O}_{7}$. In $\mathrm{YBa}_{2} \mathrm{Cu}_{3} \mathrm{O}_{7}$, the uniform spin susceptibility, $\chi_{0}$, is essentially temperature independent, and the product ${ }^{63} T_{1} T$ of the nuclear spin-lattice relaxation time and the temperature has monotonic temperature dependence; in the underdoped compounds $\chi_{0}$ is temperature dependent while the temperature dependence of ${ }^{63} T_{1} T$ is non-monotonic. Recent measurements of the spin-echo decay time, $T_{2 \mathrm{G}}$ [1], for $\mathrm{YBa}_{2} \mathrm{Cu}_{3} \mathrm{O}_{6.63}$ [2], together with the earlier measurements for $\mathrm{YBa}_{2} \mathrm{Cu}_{3} \mathrm{O}_{6.9}$ [3], provide additional valuable information on such spin gap phenomena in the underdoped systems.

Alternative physical origins of the spin gap have been proposed recently by Millis and Monien [4] and Sokol and Pines [5], hereafter SP. Millis and Monien [4] argued on the basis of bulk susceptibility measurements that the spin gaps in $\mathrm{YBa}_{2} \mathrm{Cu}_{3} \mathrm{O}_{6.63}$ and $\mathrm{La}_{1.85} \mathrm{Sr}_{0.15} \mathrm{CuO}_{4}$ possess a different physical origin, with the gap in $\mathrm{YBa}_{2} \mathrm{Cu}_{3} \mathrm{O}_{6.63}$ arising from a magnetic coupling between adjacent $\mathrm{CuO}_{2}$ layers, while for $\mathrm{La}_{1.85} \mathrm{Sr}_{0.15} \mathrm{CuO}_{4}$ it is attributed to the spin density wave fluctuations of a metal. On the other hand, SP applied scaling arguments to the analysis of the NMR experiments to determine the magnetic phase diagram of the cuprates. They argued that at high temperatures these materials, and the closely related system, $\mathrm{YBa}_{2} \mathrm{Cu}_{4} \mathrm{O}_{8}$, are in the quantum critical (QC), $\mathrm{z}=1$, regime (see [6,7]), characterized by a temperature independent ratio ${ }^{63} \mathrm{~T}_{1} \mathrm{~T} / \mathrm{T}_{2} \mathrm{G}$, and a linear dependence on $\mathrm{T}$ of ${ }^{63} \mathrm{~T}_{1} \mathrm{~T}$ [8:7], while the common physical origin of the spin gap behavior these systems exhibit at lower temperatures is the suppression of the spectral weight for frequencies, $\omega<\Delta \sim \mathrm{c} / \xi$, characteristic of the quantum disordered (QD) regime. In the quantum critical regime [6.]7], $\bar{\omega} \sim \xi^{-1} \sim T$ and $z=1$, while in the quantum disordered regime $\xi$ saturates ( $\xi$ is the antiferromagnetic correlation length, $\bar{\omega}$ characteristic energy scale, and $z$ dynamical exponent). In the present communication we show that the spin susceptibility and neutron scattering measurements on these underdoped cuprates are consistent with the SP magnetic phase diagram, reconcile the low frequency spin dynamics measured by NMR with the results of neutron scattering experiments, and give estimates of the spin gap, $\Delta$, the spin 
wave velocity, c, and $\xi$.

We consider first the Knight shift and bulk susceptibility measurements. As shown in Ref. [7], in the QC, $z=1$, regime, $\chi_{0}$ is linear in $T$, in agreement with numerical calculations 9] as well as experiment for $\mathrm{La}_{2} \mathrm{CuO}_{4}$. In the renormalized classical regime, $\chi_{0}$ is finite at $T=0$; on the other hand, in the QD regime, the susceptibility rapidly (exponentially for the insulator) decreases due to the presence of the gap in the spin excitation spectrum, and tends to zero as $T \rightarrow 0$ [7]. As may be seen in Fig.1, at high temperatures $\chi_{0}(T)$ displays the expected linear in $\mathrm{T}$ behavior for $\mathrm{La}_{2} \mathrm{CuO}_{4}, \mathrm{La}_{1.85} \mathrm{Sr}_{0.15} \mathrm{CuO}_{4}, \mathrm{YBa}_{2} \mathrm{Cu}_{3} \mathrm{O}_{6.63}$, and $\mathrm{YBa}_{2} \mathrm{Cu}_{4} \mathrm{O}_{8}$ with somewhat varying slope.

The crossover from the $\mathrm{QC}$ to the $\mathrm{QD}$ regime is clearly visible in the experimental data shown in Fig.1 1 , apart from the insulator. As discussed in SP, the temperature $\mathrm{T}^{*}$, at which ${ }^{63} \mathrm{~T}_{1} \mathrm{~T}$ starts to deviate from its linear behavior $\left(\mathrm{T}^{*} \sim 200 \mathrm{~K}\right.$ for $\mathrm{YBa}_{2} \mathrm{Cu}_{3} \mathrm{O}_{6.63}$ and $\mathrm{YBa}_{2} \mathrm{Cu}_{4} \mathrm{O}_{8}$, and $\mathrm{T}^{*} \sim 125 \mathrm{~K}$ for $\mathrm{La}_{1.85} \mathrm{Sr}_{0.15} \mathrm{CuO}_{4}$ ), signals the beginning of this crossover. This downturn, when $\chi_{0}$ rapidly (although not exponentially in the doped case) decreases for $T \lesssim \Delta$, reflects the suppression of the low frequency spin excitations. As may be seen in Fig.11, the downturn in $\chi_{0}(T)$ begins at nearly the same temperature as that at which ${ }^{63} T_{1} T$ begins to deviate from its linear behavior. It follows that, as expected for the $\mathrm{QD}$ regime, the spin gap influences the low frequency dynamics both near $\mathbf{q}=0$ and $\mathbf{q}=(\pi, \pi)$. The experimental finding that the ${ }^{17} \mathrm{O}$ spin lattice relaxation rate in $\mathrm{YBa}_{2} \mathrm{Cu}_{3} \mathrm{O}_{6.63}$ 10 and $\mathrm{YBa}_{2} \mathrm{Cu}_{4} \mathrm{O}_{8}\left[11\right.$ follows the temperature dependence of $\chi_{0}(\mathrm{~T})\left[\left({ }^{17} \mathrm{~T}_{1} \mathrm{~T}\right)^{-1} \sim \chi_{0}(\mathrm{~T})\right]$ is thus consistent with our scenario. If one assumes, with SP, that the effect of the quasiparticles on the QNL $\sigma$ model parameters is somewhat less for $\mathrm{La}_{1.85} \mathrm{Sr}_{0.15} \mathrm{CuO}_{4}$ than in $\mathrm{YBa}_{2} \mathrm{Cu}_{3} \mathrm{O}_{6.63}$, and ascribes this to a smaller hole density in the former material, and also, as Dupree et al. [12 argue, that $\mathrm{YBa}_{2} \mathrm{Cu}_{4} \mathrm{O}_{8}$ possesses a hole density comparable to that found in $\mathrm{YBa}_{2} \mathrm{Cu}_{3} \mathrm{O}_{6.8}$, we see that the temperature at which the downturn occurs increases with hole concentration, going from $\sim 100 \mathrm{~K}$ for $\mathrm{La}_{1.85} \mathrm{Sr}_{0.15} \mathrm{CuO}_{4}$ to $\sim 240 \mathrm{~K}$ for $\mathrm{YBa}_{2} \mathrm{Cu}_{3} \mathrm{O}_{6.63}$ and $\mathrm{YBa}_{2} \mathrm{Cu}_{4} \mathrm{O}_{8}$. Moreover, since the magnitude of $\chi_{0}(\mathrm{~T})$ in the $\mathrm{QC}$ and QD regime may be expected to increase with hole doping, this trend would be likewise evident in Fig.11, once 
one assigns a hole density in $\mathrm{La}_{1.85} \mathrm{Sr}_{0.15} \mathrm{CuO}_{4}$ similar to that found for $\mathrm{YBa}_{2} \mathrm{Cu}_{3} \mathrm{O}_{6.55}$, and takes its orbital Knight shift to be in the range $0.02 \%$ to $0.076 \%$.

A close examination of the data shows that two successive downturns are visible in Fig.1, with only the second corresponding to the superconducting transition (Fig.11). This supports the view that in the superconducting state of QD materials, two separate gaps are present; a charge gap which reflects the pairing of quasiparticles in the superconducting state, and a spin gap $\Delta \sim 1 / \xi$, which reflects the QD behavior modified, in the superconducting state, by the pairing correlations.

We turn next to the evidence for a spin gap and a cross-over from the QC to the QD regime found in the neutron scattering experiments on the $\mathrm{YBa}_{2} \mathrm{Cu}_{3} \mathrm{O}_{6+x}$ system. Consider first $\mathrm{YBa}_{2} \mathrm{Cu}_{3} \mathrm{O}_{6.6}$, where the measurements of Ref. [13 show that the q-integrated (i.e. local) dynamical response function, $\chi_{L}^{\prime \prime}(\omega)=\int d^{2} \mathbf{q} \chi^{\prime \prime}(\mathbf{q}, \omega)$, is a universal function of $\omega / T$ 113 which behaves as $\chi_{L}^{\prime \prime} \sim \omega / T$ for high temperatures (i.e. small $\omega / T$ ). This behavior is consistent with the QC scaling found for this compound in the analysis of the NMR experiments [5]. Moreover, the value of $\bar{\chi}_{L}(\bar{\omega})=T \chi_{L}(\omega)$ inferred from experiment [14] is in good agreement with the universal function calculated using the $1 / N$ expansion [7]. For low temperatures, the suppression of $\chi_{L}^{\prime \prime}$ for $\mathrm{YBa}_{2} \mathrm{Cu}_{3} \mathrm{O}_{6.63}$, which is seen as an abrupt deviation from the universal behavior of $\chi_{L}^{\prime \prime}$ as a function of $\omega / T$, is an indication of the spin gap phenomenon. From an analysis of their experimental results, Tranquada et al. [13] conclude that the magnitude of the spin gap for this material is $\Delta \simeq 10 \mathrm{meV}$.

Since at $T=0, \chi_{L}^{\prime \prime}(\omega)=0$ for $\omega<\Delta$, an alternative approach is to associate the maximum of $d \chi_{L}^{\prime \prime}(\omega) / d \omega$ with the spin gap magnitude, $\Delta$. Rossat-Mignod et al. [15] find that for $\mathrm{YBa}_{2} \mathrm{Cu}_{3} \mathrm{O}_{6.69}$ this maximum occurs at $16 \mathrm{meV}$ and we take this to be the value of $\Delta$ for this compound. At lower hole doping, in the range $0.4<x<0.5$ just above the metalinsulator transition at $\mathrm{x} \cong 0.41$, according to Ref. [5], $\mathrm{YBa}_{2} \mathrm{Cu}_{3} \mathrm{O}_{6+x}$ is expected to be in the QC region for $T \geq 50 \mathrm{~K}$. While no spin gap was seen in the non-superconducting $\mathrm{YBaCuO}_{6.4}$, Rossat-Mignod et al. [15] have reported the existence of a small gap in the low temperature superconducting region of $\mathrm{YBaCuO}_{6.45}$ and $\mathrm{YBaCuO}_{6.51}$. 
At higher hole doping, in the heavily-doped $\mathrm{YBa}_{2} \mathrm{Cu}_{3} \mathrm{O}_{6+x}$ with $x \simeq 1$, a value of the spin gap $\sim 26 \mathrm{meV}$ has been reported by Rossat-Mignod et al. using unpolarized neutrons [15]. Mook et al. [16] have used polarized neutrons to study $\mathrm{YBa}_{2} \mathrm{Cu}_{3} \mathrm{O}_{7}$ and have reported that a low-frequency suppression of the signal, ascribed to the spin gap formation, may be present at $\sim 35 \mathrm{meV}$; they find as well a magnetic peak at $41 \mathrm{meV}$. Inelastic neutron experiments in the intermediate region of oxygen concentrations 6.75 and 6.9 have also been reported in Ref. [14, but the measurements have been performed only at low temperatures, where the superconducting gap, which complicates such an analysis and may affect the spin gap, is already developed. We show in Fig.2 the values of $\Delta$ which have been reported for various oxygen concentrations.

Our conclusion that the spin gap increases as the doping increases is somewhat unexpected in view of previous analyses [17], but may be easily understood in terms of the QNL $\sigma$ model, where $\Delta=c / \xi$, provided $\xi$ increases faster than c as the doping decreases. One can, moreover, combine the results of neutron scattering and NMR experiments to obtain an estimate of the doping dependence of $\mathrm{c}$ and $\xi$.

We consider first $\mathrm{YBa}_{2} \mathrm{Cu}_{3} \mathrm{O}_{6.63}$, for which we estimate $\Delta \cong 12 \mathrm{meV}$ by interpolating between the experimental results for $\mathrm{YBa}_{2} \mathrm{Cu}_{3} \mathrm{O}_{6.6}$ and $\mathrm{YBa}_{2} \mathrm{Cu}_{3} \mathrm{O}_{6.69}$. This value is somewhat less than that found by applying scaling arguments to the experimental results for $T_{2 G}(T)$, shown in Fig. 娄. Applying the approach of [7], one obtains that in the QC regime, $T_{2 G}(T)$ is linear in $\mathrm{T}$, while the ratio of this slope and its extrapolated intercept is the product of a universal number, $\zeta$, and $\Delta$. On taking $\zeta$ as obtained from the leading order of a $1 / \mathrm{N}$ expansion, one finds $\Delta=18 \mathrm{meV}$; the discrepancy between the two results plausibly reflects next-order corrections to $\zeta$, as well as uncertainties in the determination of $\Delta$ in a neutron scattering experiment.

A different approach is to make use of the scaling result $\chi_{Q} \sim \xi^{2-\eta}, \eta \ll 1$, and neglect any possible temperature dependence of $\alpha$ in $\chi_{\mathrm{Q}}=\alpha \xi^{2}$. To the extent this holds, the full temperature dependence of $\xi(\mathrm{T})$ may be determined directly from $T_{2 G}$, as shown in Fig. [3. Moreover, to the extent that the QNL $\sigma$ model is valid, despite the absence of a rigorous 
Lorentz invariance of the action, the relation $\Delta=\mathrm{c} / \xi$ should still approximately apply in the $\mathrm{QD}$ regime at low temperatures. With these assumptions, for a given choice of $\alpha, \chi_{\mathrm{Q}}$, $\xi$, and c may be estimated by comparing the neutron scattering result $\Delta \cong 12 \mathrm{meV}$ with the NMR results for ${ }^{63} \mathrm{~T}_{1}$ and $\mathrm{T}_{2 \mathrm{G}}$ and assuming Lorentzian form of $\chi(\widetilde{q})$. For convenience, we introduce a dimensionless quantity $\bar{\alpha}_{\mathrm{TP}}=\alpha / \alpha_{\mathrm{TP}}$, where $\alpha_{\mathrm{TP}}=14.8$ states $/ \mathrm{eV}$ is a value of $\alpha$ estimated by Thelen and Pines [18] for $\mathrm{YBa}_{2} \mathrm{Cu}_{3} \mathrm{O}_{6.9}$. We find that at low temperatures, in the $\mathrm{QD}$ regime, $\xi \simeq 4.2 \bar{\alpha}_{\mathrm{TP}}^{-1}$ and $\mathrm{c} \simeq \bar{\alpha}_{\mathrm{TP}}^{-1} \cdot 0.19 \mathrm{eV} \AA$, while in the $\mathrm{QC}$ regime, one has $\xi^{-1}=\bar{\alpha}_{\mathrm{TP}} \cdot 0.1(1+\mathrm{T} / 100 \mathrm{~K})$. We note in passing that T-linear QC behavior of both $\xi^{-1}$ and $T_{2 \mathrm{G}}$ in a doped antiferromagnet has been recently reported by Glenister, Singh, and one of us (A.S.) [19]; see also Ref. [7].

We examine next the extent to which the neutron scattering experimental results on $\mathrm{La}_{1.85} \mathrm{Sr}_{0.15} \mathrm{CuO}_{4}$ are consistent with the NMR results, the magnetic phase diagram of SP, and our proposed variation of $\Delta$ with hole concentration. Here there are two issues. First, if the hole doping in $\mathrm{La}_{1.85} \mathrm{Sr}_{0.15} \mathrm{CuO}_{4}$ is comparable to that found in $\mathrm{YBa}_{2} \mathrm{Cu}_{3} \mathrm{O}_{6.55}$, one would expect from Fig.2 that the spin gap $\Delta$ for $\mathrm{La}_{1.85} \mathrm{Sr}_{0.15} \mathrm{CuO}_{4}$ is $\sim 6 \mathrm{meV}$. While the experiments reported in Ref. [20] show no clear evidence for such a gap, we find an upturn near $\omega \leq 6 \mathrm{meV}$ in $\chi_{\mathrm{L}}^{\prime \prime}(\omega)$ at $35 \mathrm{~K}$ measured by Thurston et al. [21], as might be expected if $\Delta \sim 6 \mathrm{meV}$ for this material. Second, while the incommensurate spin fluctuation peaks, at $(\pi, \pi \pm \delta)$ and $(\pi \pm \delta, \pi)$, where $\delta=0.245 \pi$, reported in Ref. [20], are not incompatible with our picture, such a large value of $\delta$ is incompatible with NMR experiments. The results of Ref. [22] confirm the prediction [23] that the hyperfine constants for the 214 systems are quite close to those inferred for the 123 systems and specifically, for $\mathrm{YBa}_{2} \mathrm{Cu}_{3} \mathrm{O}_{6.63}$ [24]. On taking the neutron results for $\chi^{\prime \prime}(\mathbf{q}, \omega)$ it is straightforward to show that at $100 \mathrm{~K}$, the copper-to-oxygen relaxation rate ratio, $\left({ }^{17} \mathrm{~T}_{1} /{ }^{63} \mathrm{~T}_{1, \|}\right) \lesssim 35$ while the ${ }^{63} \mathrm{Cu}$ relaxation rate anisotropy ratio, $\left({ }^{63} T_{1, \|} /{ }^{63} T_{1, \perp}\right) \sim 4.5$. These results, which reflect the leakage of correlations away from $(\pi, \pi)$, brought about by the comparatively large value of $\delta$, are to be compared to those found experimentally: $\left({ }^{17} \mathrm{~T}_{1, \|} /{ }^{63} \mathrm{~T}_{1, \|}\right) \gtrsim 80$ [25] and $\left({ }^{63} \mathrm{~T}_{1, \|} /{ }^{63} \mathrm{~T}_{1, \perp}\right) \sim 2.6$.

Can the NMR and neutron scattering results be reconciled? The problem is not with the 
magnitude of the measured spin fluctuation peaks. The value of ${ }^{63} \mathrm{~T}_{1, \|} \mathrm{T}$ is $\sim 5.5 \times 10^{-2} \mathrm{~K} \cdot \mathrm{sec}$ at $\mathrm{T}=35 \mathrm{~K}$, when calculated from the neutron scattering results of Ref. 20 for q-integrated intensity $\chi_{\mathrm{L}}(\omega)$, assuming commensurability. This compares favorably with the NMR result, ${ }^{63} \mathrm{~T}_{1, \|} \mathrm{T} \sim 5.9 \cdot 10^{-2} \mathrm{~K} \cdot \mathrm{sec}$; indeed the slightly larger value at $35 \mathrm{~K}$ may reflect the continued upturn associated with a spin gap $\sim 6 \mathrm{meV}$. The problem rather lies in the assumption that the peaks are incommensurate. If instead, as suggested independently by Slichter [26] and Phillips [27] what is being observed is discommensuration, associated with the formation of domains in the $\mathrm{Cu}-\mathrm{O}$ plane, the NMR and neutron scattering experiments can be reconciled, as shown in recent calculations by Monthoux [28]. Thus in neutron scattering one is likely seeing both commensurate spin fluctuation peaks and domain structure. Two other experimental results lend support to this proposal: the appearance of a slightly incommensurate peak in $\mathrm{YBa}_{2} \mathrm{Cu}_{3} \mathrm{O}_{6.6}$ [13] which might be expected to show behavior not very different from that of $\mathrm{La}_{1.85} \mathrm{Sr}_{0.15} \mathrm{CuO}_{4}$, and the appearance of a second ${ }^{63} \mathrm{Cu}$ resonance line in both the Sr-doped 214 systems [29] and $\mathrm{La}_{2} \mathrm{CuO}_{4.032}$ [30], which would plausibly be associated with ${ }^{63} \mathrm{Cu}$ nuclei located in or near domain walls.

In summary, we have shown that NMR, bulk susceptibility, and neutron scattering data in the underdoped materials are consistent with the picture of Sokol and Pines [5], where the spin gap onset is associated with the crossover from the $\mathrm{QC}, z=1$, regime to the low temperature QD regime. The spin gap magnitudes in $\mathrm{YBa}_{2} \mathrm{Cu}_{3} \mathrm{O}_{6.63}, \mathrm{YBa}_{2} \mathrm{Cu}_{4} \mathrm{O}_{8}$, and $\mathrm{La}_{1.85} \mathrm{Sr}_{0.15} \mathrm{CuO}_{4}$ compounds determined from different experiments are consistent with each other. The magnitude of the spin gap gradually increases as doping increases; since the quasiparticle contribution also increases, this eventually leads to the smearing of the gap and a crossover to the overdamped, $z=2$, regime in the fully doped materials. Such a doping dependence is consistent with the theoretical prediction that $\Delta \sim c / \xi$, provided the correlation length decreases more rapidly then $\mathrm{c}$ as the doping increases.

We are grateful to P.C. Hammel, T. Imai, T.E. Mason, A.J. Millis, R.R.P. Singh, C.P. Slichter, and M. Takigawa for many stimulating discussions, to T. Imai, C.P. Slichter, and M. Takigawa for communicating their experimental data prior to publication, and to G. 
Aeppli and T.E. Mason for cooperation in making comparisons between the NMR and neutron scattering data. This work was supported by the NSF Grant DMR89-20538 through the Materials Research Laboratory and DMR91-20000 through the Science and Technology Center for Superconductivity. 


\section{REFERENCES}

[1] C.H. Pennington and C.P. Slichter, Phys. Rev. Lett. 66, 381 (1991); see also T. Imai, J. Phys. Soc. Japan 59, 2508 (1990).

[2] M. Takigawa, unpublished.

[3] T. Imai et al., Phys. Rev. B 47, 9158 (1993).

[4] A.J. Millis and H. Monien, Phys. Rev. Lett. 70, 2810 (1993).

[5] A. Sokol and D. Pines, preprint.

[6] S. Chakravarty, B.I. Halperin, and D.R. Nelson, Phys. Rev. B 39, 2344 (1989).

[7] A.V. Chubukov and S. Sachdev, Phys. Rev. Lett. 71, 169 (1993). A.V. Chubukov, S. Sachdev, and J. Ye, preprint (1993); see also S. Sachdev and J. Ye, Phys. Rev. Lett. 69, 2411 (1992).

[8] S. Chakravarty and R. Orbach, Phys. Rev. Lett. 64, 224 (1990).

[9] R.L. Glenister and R.R.P. Singh, preprint.

[10] M. Takigawa et al., Phys. Rev. B 43, 247 (1991).

[11] G.-Q. Zheng et al., Physica C 193, 154 (1992).

[12] R. Dupree et al., Physica C 179, 311; ibid., 185\&189, 1219 (1991).

[13] J. M. Tranquada et al., Phys. Rev. B 46, 5561 (1992); B. J. Sternlieb et al., Phys. Rev. B 47, 5320 (1993).

[14] M. Sato et al., J. Phys. Soc. Japan 62, 263 (1993).

[15] J. Rossat-Mignod et al., Physica Scripta T45, 74 (1992); J. Rossat-Mignod et al., Physica B 180\&181, 383 (1992) and references therein.

[16] H. A. Mook et al., Phys. Rev. Lett. 70, 3490 (1993). 
[17] A.J. Millis, preprint (1993), and references therein.

[18] D. Thelen and D. Pines, preprint (1993).

[19] A. Sokol, R.L. Glenister, and R.R.P. Singh, unpublished.

[20] S.-W.Cheong et al., Phys. Rev. Lett. 67, 1791 (1991); T.E.Mason, G.Aeppli, and H.A.Mook, Phys. Rev. Lett. 68, 1414 (1992); G.Aeppli and T.E.Mason, private communication.

[21] J. R. Thurston et al., Phys. Rev. B 46, 9128 (1992).

[22] A. Sokol, E. Gagliano, and S. Bacci, Phys. Rev. B 47, 14646 (1993); and unpublished.

[23] T. Imai and C.P. Slichter, private communication.

[24] H. Monien, D. Pines, and M. Takigawa, Phys. Rev. B 43, 258 (1991).

[25] L. Reven et al., Phys. Rev. B 43, 10466 (1991).

[26] C.P. Slichter, private communication.

[27] J.C. Phillips, Solid State Commun. 84, 189 (1992).

[28] P. Monthoux, unpublished.

[29] K. Yoshimura et al., J. Phys. Soc. Japan 58, 3057 (1989).

[30] P.C. Hammel et al., Phys. Rev. Lett., in press.

[31] D.C. Johnston et al., in Electronic Properties and Mechanisms of High-T $T_{c}$ Superconductors, Eds. T. Oguchi et al., Elsevier (1992).

[32] K. Ishida, Y. Kitaoka, G. Zheng, K. Asayama, Journ. Phys. Soc. Japan, 60, 3516 (1991).

[33] T. Machi et al., Physica C 173, 32 (1991). 


\section{FIGURES}

FIG. 1. The spin susceptibility for: $\square-\mathrm{La}_{2} \mathrm{CuO}_{4}$ [31], from the bulk susceptibility; $\mathrm{La}_{1.85} \mathrm{Sr}_{0.15} \mathrm{CuO}_{4}$ [32], from $\mathrm{Cu}$ Knight shift; - $-\mathrm{YBa}_{2} \mathrm{Cu}_{3} \mathrm{O}_{8}$ [33], from Cu Knight shift; O ( $\left.{ }^{63} \mathrm{~K}_{a b}\right)$, $\triangle\left({ }^{17} \mathrm{~K}_{a x}\right), \nabla\left({ }^{17} \mathrm{~K}_{c}\right), \diamond\left({ }^{17} \mathrm{~K}_{\text {iso }}\right)-\mathrm{YBa}_{2} \mathrm{Cu}_{3} \mathrm{O}_{6.63}$ [10, from the Knight shift. Solid lines show linear fits to the high temperature parts of the respective data, except for $\mathrm{YBa}_{2} \mathrm{Cu}_{3} \mathrm{O}_{6.63}$, where the slope could not be determined because little high temperature data is available; it has been chosen to be the same as for $\mathrm{YBa}_{2} \mathrm{Cu}_{4} \mathrm{O}_{8}$ instead.

FIG. 2. The values of the spin gap in $\mathrm{YBa}_{2} \mathrm{Cu}_{3} \mathrm{O}_{y}$ at different oxygen concentrations $y$ determined in inelastic neutron scattering experiments, see the text for discussion; $\mathbf{\square}$ - M. Sato et al. [14], $\diamond$ - J. Rossat-Mignod et al [15], $\Delta$ - J. M. Tranquada et al [13], O - H. A. Mook et al [16].

FIG. 3. The Gaussian spin-echo decay time for $\mathrm{YBa}_{2} \mathrm{Cu}_{3} \mathrm{O}_{6.63}$, after Ref. [2]. The solid line is the high temperature linear fit (QC regime); the dotted line represents its saturation in the QD regime. An alternate axis shows the temperature dependence of $\xi^{-1}$ as given by the scaling relation $T_{2 \mathrm{G}} \sim 1 / \xi$ 